\title{
High-speed Chemical Imaging System Based on Front-side-illuminated LAPS
}

\author{
Akinori Itabashi ${ }^{1}$, Naoki Kosaka ${ }^{1}$, Ko-ichiro Miyamoto ${ }^{1}$, Torsten Wagner ${ }^{1}$, Tatsuo Yoshinobu ${ }^{1,2}$ \\ ${ }^{1}$ Department of Electronic Engineering, ${ }^{2}$ Department of Biomedical Engineering, Tohoku University \\ 2-1-1 Katahira, Aoba-ku, Sendai, 980-8577, Japan \\ itabashi@ecei.tohoku.ac.jp
}

\begin{abstract}
The chemical imaging sensor is a Si-based chemical sensor that visualizes the spatial distribution of a specific ion in the solution contacted with the sensing surface. It is based on the principle of the lightaddressable potentiometric sensor (LAPS), in which a modulated light beam is used to read out the ion concentration in the form of photocurrent. Since the conventional chemical imaging sensor uses a scanning light beam for mapping the distribution of the ion concentration, the long scan time has been a problem for imaging in real time. For high-speed imaging, a plurality of light beams modulated at different frequencies can be employed to measure the ion concentrations simultaneously at different locations. Although a wide frequency bandwidth is required for high-speed imaging, the modulation frequency is limited due to the low-pass characteristics of carrier diffusion in the conventional setup, in which the back-side of the Si substrate is illuminated. In this study, a high-speed chemical imaging system based on front-side-illuminated LAPS was developed, which achieved imaging of $\mathrm{pH}$ distribution at 70 frames per second.
\end{abstract}

Key words: light-addressable potentiometric sensor (LAPS), chemical imaging sensor, EIS, and pH

\section{Introduction}

The light-addressable potentiometric sensor (LAPS) [1] is a Si-based chemical sensor with an electrolyte-insulator-semiconductor (EIS) structure, in which the $\mathrm{Si}$ substrate is illuminated with a modulated light and the induced $A C$ photocurrent is measured to determine the ion concentration on the sensing surface. Here, the measurement area can be defined by illumination. The chemical imaging sensor [2] is based on the addressability of LAPS, in which a scanning light beam is used to obtain a 2-D map of the ion concentration, but it was difficult to observe the dynamics of chemical reaction in real time because of the slow scan rate.

For high-speed imaging, a plurality of light beams modulated at different frequencies can be employed to read out the ion concentrations at different locations in parallel $[3,4]$ as shown in Fig.1. In this case, the photocurrent signal is a superposition of all frequency components, which can be separated by Fourier analysis. Here, the required bandwidth of the modulation frequency is given by (number of light beams) $x$ (frequency spacing) and the time window of Fourier analysis must be at least as long as 1/ (frequency spacing). This means that highspeed imaging requires a wide bandwidth of the modulation frequency. Due to the low-pass characteristics of carrier diffusion across the sensor plate, however, the modulation frequency is limited in the conventional setup, where the back-side of the Si substrate is illuminated. For front-side illumination, on the other hand, photocarriers are generated and separated directly inside the depletion layer and higher frequencies can be used [5]. In this study, a high-speed chemical imaging system based on front-side-illuminated LAPS was developed using a 2-D array of LEDs.

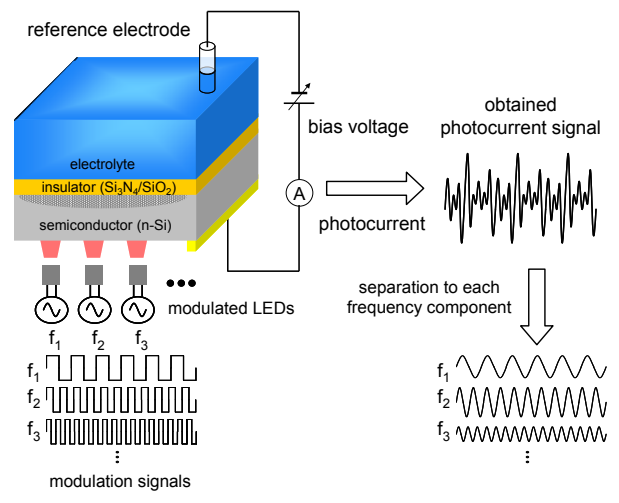

Fig.1 Diagram of simultaneous measurement. 


\section{Front-side vs. Back-side Illumination}

Two different geometries are possible in the measurement of LAPS. In the front-side and back-side illumination, the front-side or the back-side of the Si substrate is illuminated by the modulated light, respectively [5].

As shown in Fig.2 (a), the photocurrent generated by back-side illumination is smaller than that of front-side illumination, due to recombination of photocarriers in the course of diffusion across the sensor plate.

In addition, the decrease of photocurrent with the modulation frequency in back-side illumination is steeper than that of front-side illumination as shown in Fig. 2 (b).

This result suggests that front-side illumination is more advantageous for high-speed chemical imaging, for which higher modulation frequencies are required.

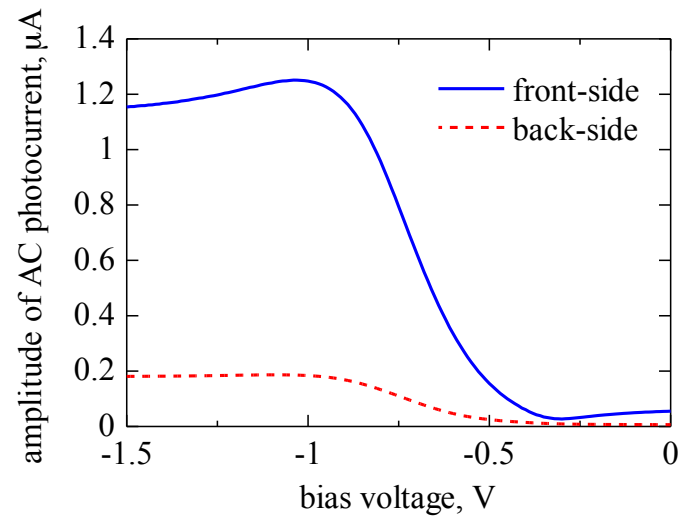

(a)

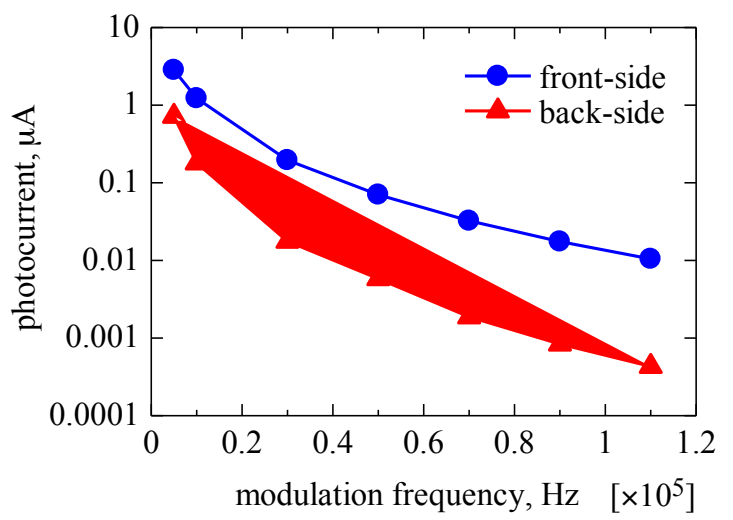

(b)

Fig.2 (a) I-V curves for front-side and backside illumination (LED light modulated at $10 \mathrm{kHz}$ ), and (b) magnitude of photocurrent as a function of modulation frequency for front-side and back-side illumination.

\section{High-speed Chemical Imaging System}

Fig.3 (a) shows a schematic view of the highspeed chemical imaging system based on frontside-illuminated LAPS. The system consists of a sensor plate, an oscillator array to synthesize a series of modulation frequencies, a 2-D array of LEDs (LED matrix) and a control PC.

Sensor plate: The sensor plate is an n-type $\mathrm{Si}$ wafer. The top surface of the sensor plate was covered with $\mathrm{Si}_{3} \mathrm{~N}_{4}$ as a $\mathrm{pH}$-sensitive layer on the insulating layer of $\mathrm{SiO}_{2}$.

Oscillator array: Five modulation frequencies $(6,7,8,9$ and $10 \mathrm{kHz})$ are generated by 5 channel phase-locked loop (PLL) synthesizer.

LED matrix: Fig.3 (b) shows a top view of the LED matrix. The matrix has $35(5 \times 7)$ LEDs with a separation of $2.5 \mathrm{~mm}$. Five LEDs on one line are modulated at different frequencies, and five points on the sensor surface are measured simultaneously as shown in Fig.4.

PC and Software: The program controls the bias voltage, selects a line of the LED matrix in sequence, measures the photocurrent signal, calculates the amplitude of the signal at each frequency and generates a chemical image.

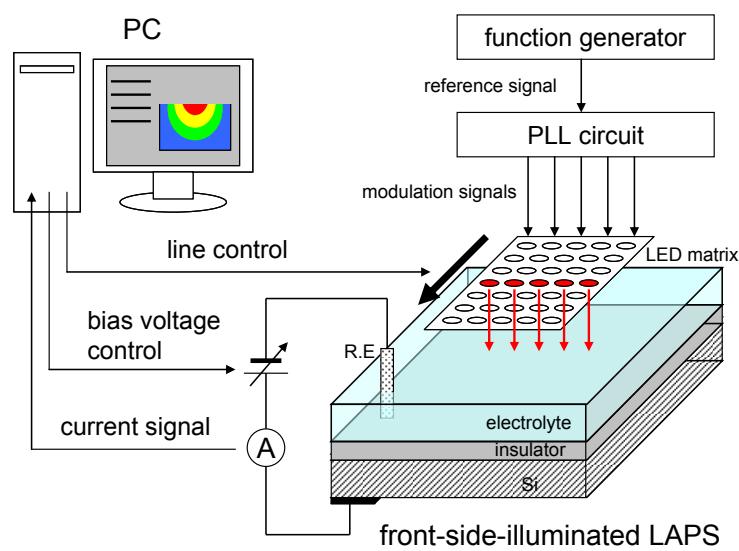

(a)

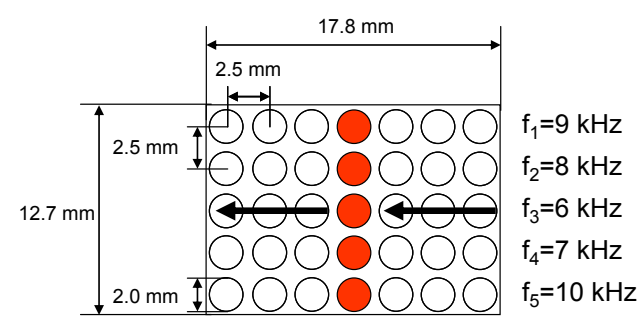

(b)

Fig.3 (a) Schematic view of the high-speed chemical imaging system based on front-side-illuminated LAPS, and (b) top view of the $L E D$ matrix. 


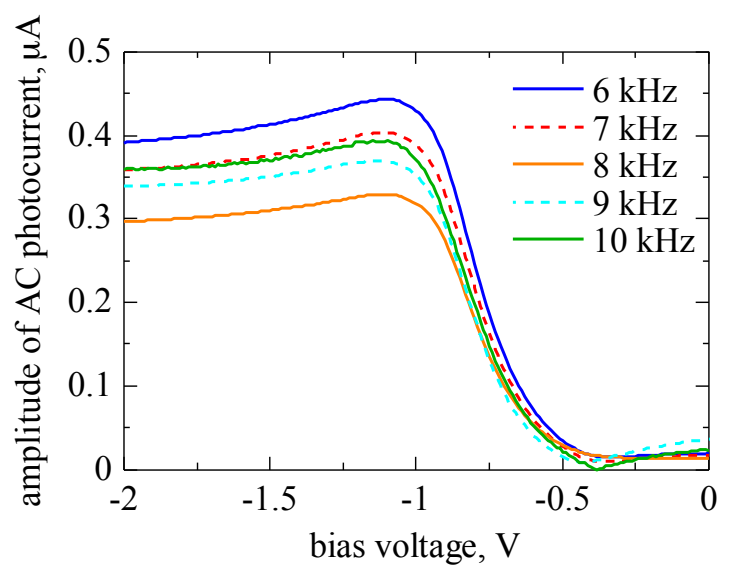

Fig.4 I-V curves for simultaneous measurement on one line of the $L E D$ matrix.

\section{Experimental Results of Chemical Imaging}

Using the developed chemical imaging system based on front-side-illuminated LAPS, the experiments of chemical imaging were performed to observe the temporal change of $\mathrm{pH}$ distribution caused by injection of a solution.

In each measurement, the photocurrent values at each measurement position are normalized by the values in the initial state to compensate for the frequency-dependence of the photocurrent and the non-uniformity of the sensor plate.

\subsection{Imaging of $\mathrm{pH}$ Buffer Solution}

Chemical imaging of buffer solutions of $\mathrm{pH} 4,5$, $6,7,8,9$, and 10 was performed. The photocurrent values at each measurement position were normalized by the values of $\mathrm{pH} 7$ buffer solution. Fig.5 shows an average of normalized photocurrent at each measurement position as a function of $\mathrm{pH}$ value. It proves that the normalized photocurrent varies almost linearly with $\mathrm{pH}$ value. Fig.6 shows the experimental result of chemical imaging. As shown in Fig.6, the chemical images of each $\mathrm{pH}$ buffer solution are almost uniform in color display corresponding to the uniform $\mathrm{pH}$ distribution.

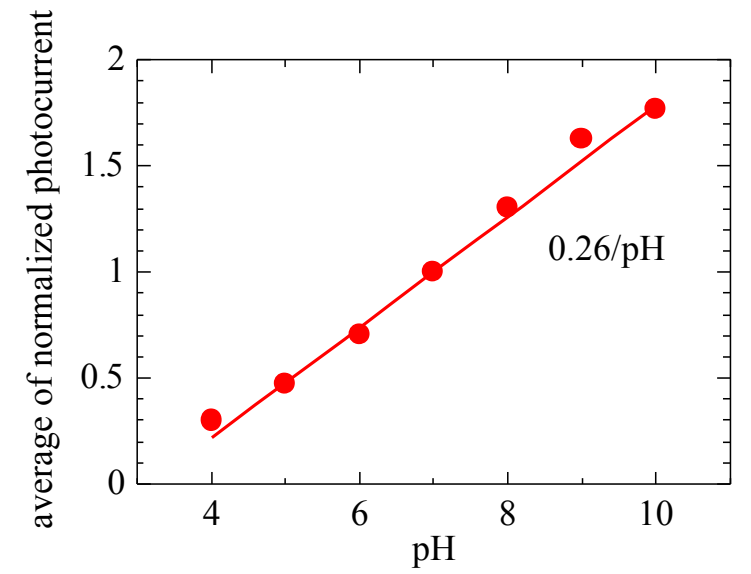

Fig.5 Average of normalized photocurrent as a function of $\mathrm{pH}$ value.

\subsection{High-speed Imaging at $14 \mathrm{fps}$}

Fig.7 shows the experimental result of highspeed chemical imaging at $14 \mathrm{fps}$ when $0.2 \mathrm{ml}$ of $0.1 \mathrm{M} \mathrm{HCl}$ solution was injected into $2.0 \mathrm{ml}$ of $0.1 \mathrm{M} \mathrm{NaCl}$ solution at a rate of $0.13 \mathrm{ml} / \mathrm{sec}$. Spreading of acidic area is observed in real time.

Fig.8 shows the experimental result of highspeed chemical imaging at $14 \mathrm{fps}$ when $0.2 \mathrm{ml}$ of $0.1 \mathrm{M} \mathrm{HCl}$ solution was injected into $2.0 \mathrm{ml}$ of neutral buffer solution at a rate of $0.13 \mathrm{ml} / \mathrm{sec}$. After spreading of acidic area, shrinking of acidic area by buffering action is observed.

\subsection{High-speed Imaging at $70 \mathrm{fps}$}

Chemical imaging at even higher speed is possible by reducing the sampling number.

Fig.9 shows the experimental result of highspeed chemical imaging at $70 \mathrm{fps}$ when $0.3 \mathrm{ml}$ of $0.1 \mathrm{M} \mathrm{HCl}$ solution was injected into $2.0 \mathrm{ml}$ of $0.1 \mathrm{M} \mathrm{NaCl}$ solution at a rate of $0.13 \mathrm{ml} / \mathrm{sec}$. The quick spreading of acidic area is observed at time resolution of $14.3 \mathrm{msec}$.

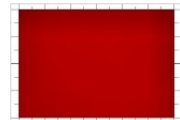

$\mathrm{pH} 4$

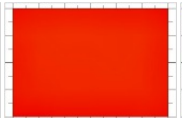

$\mathrm{pH} 5$

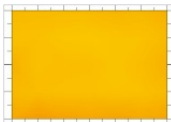

$\mathrm{pH} 6$

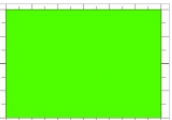

$\mathrm{pH} 7$

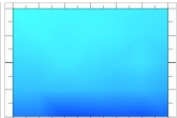

$\mathrm{pH} 8$

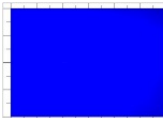

$\mathrm{pH} 9$

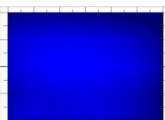

$\mathrm{pH} 10$ basic

neutral

acidic

Fig.6 Chemical images of buffer solutions of $\mathrm{pH} 4,5,6,7,8,9$, and 10. 


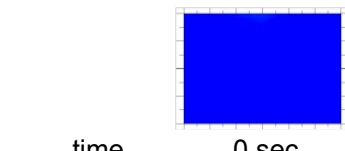

time frame number

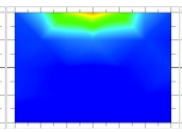

$0.4 \mathrm{sec}$

5

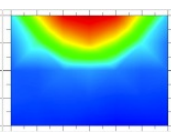

$0.7 \mathrm{sec}$

10

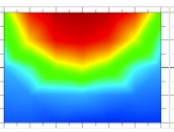

$1.1 \mathrm{sec}$

15

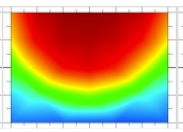

$1.8 \mathrm{sec}$ 25

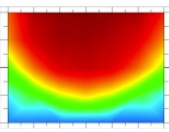

$5.7 \mathrm{sec}$

80

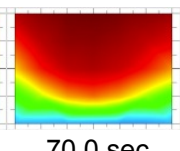

980

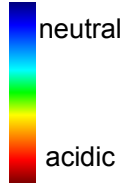

Fig.7 Example of high-speed chemical imaging at $14 \mathrm{fps}$ (injection of $\mathrm{HCl}$ in $\mathrm{NaCl}$ solution).

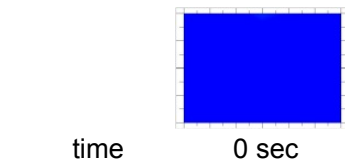

time frame number

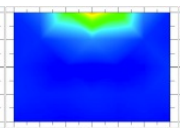

$0.7 \mathrm{sec}$

10

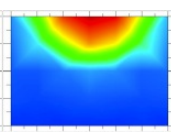

$1.4 \mathrm{sec}$

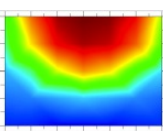

$5.7 \mathrm{sec}$

80

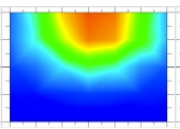

$16.4 \mathrm{sec}$ 230

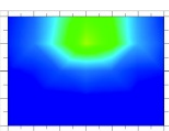

$34.3 \mathrm{sec}$ 480

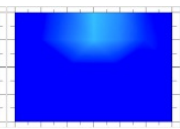

$70.0 \mathrm{sec}$ 980

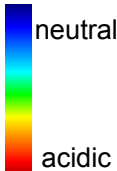

acidic

Fig.8 Example of high-speed chemical imaging at $14 \mathrm{fps}$ (injection of $\mathrm{HCl}$ in buffer solution)

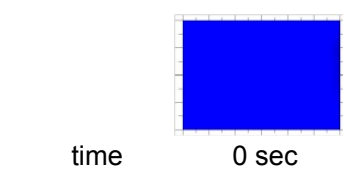

frame number

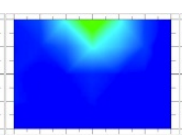

$0.29 \mathrm{sec}$

20

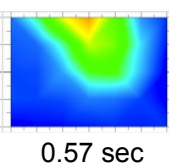

40

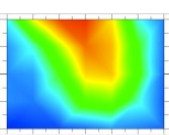

$0.86 \mathrm{sec}$

60

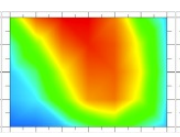

$1.14 \mathrm{sec}$

80

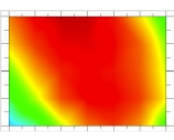

$1.72 \mathrm{sec}$

120

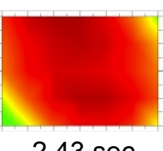

170

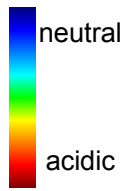

Fig.9 Example of high-speed chemical imaging at $70 \mathrm{fps}$ (injection of $\mathrm{HCl}$ in $\mathrm{NaCl}$ solution)

\section{Conclusion}

In this study, a high-speed chemical imaging system was developed based on front-sideilluminated LAPS, for which higher modulation frequencies can be used in contrast to the conventional chemical imaging system with back-side illumination. The experimental results of chemical imaging demonstrated that chemical imaging by front-side illumination is also possible. Using the developed system, the quick change of $\mathrm{pH}$ distribution could be visualized as a sequence of chemical images acquired at $70 \mathrm{fps}$ (time resolution: $14.3 \mathrm{msec}$ ) at a resolution of $5 \times 7$ pixels.

\section{References}

[1] D. G. Hafeman, J. Wallace Parce, and H. M. McConnel, Light-addressable potentiometric sensor for biochemical systems, Science 240 , 1182-1185 (1988); doi: 10.1126/science.3375810
[2] M. Nakao, T. Yoshinobu, and H. Iwasaki, Scanning-Laser-Beam Semiconductor $\mathrm{pH}$ Imaging Sensor, Sensors and Actuators B 20, 119-123 (1994); doi: 10.1016/09254005(93)01199-E

[3] Q. Zhang, P. Wang, W. J. Parak, M. George, and G. Zhang, A novel design of multi-light LAPS based on digital compensation of frequency domain, Sensors and Actuators B 73, 152-156 (2001); doi: 10.1016/S0925-4005(00)00696-1

[4] K. Miyamoto, Y. Kuwabara, S. Kanoh, T. Yoshinobu, T. Wagner, and M. J. Schöning, Chemical image scanner based on FDM-LAPS, Sensors and Actuators B 137, 533-538 (2009); doi: 10.1016/j.snb.2008.12.008

[5] M. Sartore, M. Adami, C. Nicolini, L. Bousse, S. Mostarshed, and D. Hafeman, Minority carrier diffusion length effects on light-addressable potentiometric sensor (LAPS) devices, Sensors and Actuators $A$ 32, 431-436 (1992); doi: 10.1016/0924-4247(92)80025-X 